FERMILAB-TM-2401-DO

October 2007

\title{
Accelerator/Experiment Operations - FY 2007
}

\author{
S. Brice, N. Buchanan, R. Coleman, M. Convery, D. Denisov, G. Ginther, A. Habig, \\ S. Holmes, W. Kissel, W. Lee, T. Nakaya, R. Plunkett, E. Ramberg, \\ P. Schlabach, R. Van de Water, M. Wascko, P. Wilson, D. Wood
}

Edited by J. Appel

This Technical Memorandum (TM) summarizes the Fermilab accelerator and accelerator experiment operations for FY 2007. It is one of a series of annual publications intended to gather information in one place. In this case, the information concerns the FY 2007 Run II at the Tevatron Collider, the MiniBooNE and SciBooNE experiments running in the Booster Neutrino Beam (BNB), MINOS using the Main Injector Neutrino Beam (NuMI), and the Meson Test Beam (MTest) activities in the $120 \mathrm{GeV}$ external Switchyard beam (SY120).

Each section was prepared by the relevant authors, and was somewhat edited for inclusion in this summary.

Accelerator Operations (S. Holmes)

\section{Tevatron Collider}

Tevatron Collider operations spanned the FY 2006-2007 boundary without interruption and continued until the initiation of the 2007 shutdown on August 5, 2007. The shutdown was in progress as FY 2007 came to a close, with beam commissioning startup scheduled for the third week in October, 2007. For FY 2007 (defined as 0000 on Monday morning, October 2, 2006 to 2400 on Sunday night, September 30, 2007) integrated luminosity delivered (average of CDF and DZero) totaled $1311 \mathrm{pb}^{-1}, 74 \%$ of the design goal and more than twice that delivered in FY 2006 (see Figure 1). Over 44 weeks of scheduled operations, a total of 4777 store hours was delivered, $103 \%$ of the design goal. Total delivered integrated luminosity for Run II stood at $3.207 \mathrm{fb}^{-1}$ at the end of the fiscal year.

Highlights for the year include:

- A record initial luminosity (average of CDF and DZero) of $29.2 \times 10^{31} \mathrm{~cm}^{-2} \mathrm{sec}^{-1}$ on February 25 - a 28\% increase over the record at the end of FY 2006.

- A record weekly delivered luminosity (average of CDF and DZero) of $44.8 \mathrm{pb}^{-1}$ over the period January 1-7 - a 35\% increase over the record at the end of FY 2006.

- Implementation of the new-style lithium lens, allowing operations at gradients up to 756 $\mathrm{T} / \mathrm{m}$, as compared to $560 \mathrm{~T} / \mathrm{m}$ with the old-style lens.

- An increase in the number of protons delivered from the Main Injector to the antiproton production target, culminating in a record of $8.9 \times 10^{12}$ protons per pulse averaged over twenty-four hours on July 26. 
- Improvements to the antiproton stacking rate. The record for antiprotons stacked in an hour is now $23.1 \times 10^{10}$ hour - a $15 \%$ improvement over FY 2006. The average sustainable stacking rate rose to between $21-22 \times 10^{10} /$ hour.

- Reduction of the time required to transfer antiprotons from the Accumulator to the Recycler. These transfers now routinely take 12-15 minutes.

- Improved reliability, with unscheduled downtime reduced to $17 \%$, as compared to $23 \%$ in FY 2006. This was achieved despite three weeks of unscheduled downtime due to two superconducting component failures in the first quarter of FY 2007.

\section{FY2007 Luminosity}

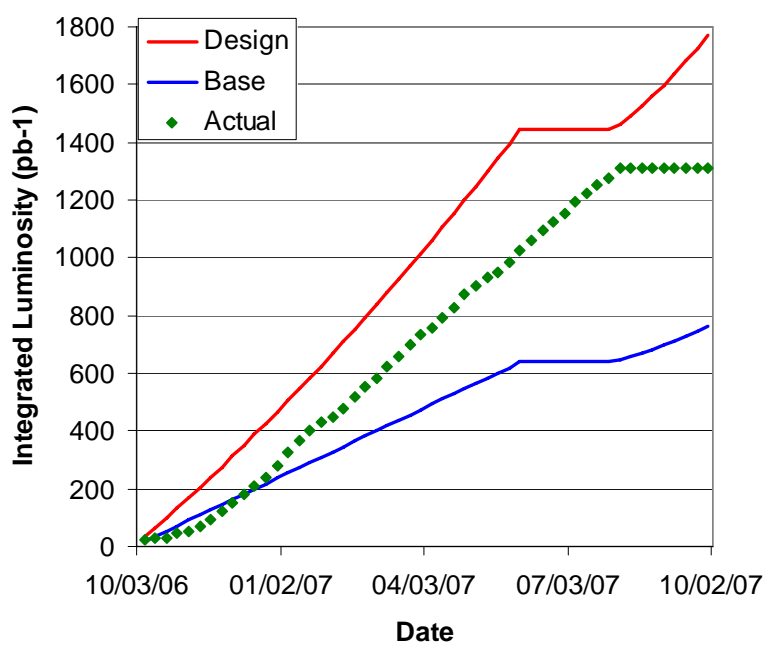

Figure 1. FY 2007 integrated luminosity, planned and delivered (average of CDF and DZero).

The stage was set for these improvements by the successful completion of the Run II Luminosity Upgrade Program at the end of FY 2006. Through FY 2007, and as planned for the balance of Run II, operations capitalized on the investment made over FY 2003-2006 to optimize running conditions and maximize integrated luminosity.

\section{Commentary on FY 2007 Collider Performance}

The median Collider luminosity over the final nine weeks of FY 2007 was $19.6 \times 10^{31} \mathrm{~cm}^{-2} \mathrm{sec}^{-1}$, a $17 \%$ increase over the end of FY 2006, and $61 \%$ of the design goal of $31.9 \times 10^{31} \mathrm{~cm}^{-2} \mathrm{sec}^{-1}$. Improved performance over the course of the year was derived from a number of sources, some anticipated and some not. The most significant factors include:

- More protons in collision

The number of protons in collision increased roughly 5\% as compared to a year ago.

Two factors pushed the proton intensity up:

- The implementation of a new $150 \mathrm{GeV}$ helix, resulting in improved proton lifetimes during antiproton loading.

- Continued performance improvements in the proton injector complex. 
- More antiprotons in collision, accompanied by good emittances

The number of antiprotons in collision has increased by roughly $10 \%$ over the last year. Primary contributors are:

- An adjustment to the tune working point in the Recycler near the end of FY 2006 was fully capitalized on in FY 2007 as operational experience was gained. This greatly improved beam lifetime and significantly reduced emittance growth during the mining process.

- The stacking rate was improved to roughly $21-22 \times 10^{10} /$ hour over the year $(5-10 \%$ improvement). The primary drivers were increases in the number of protons on target and a significant increase in the Li lens gradient.

- Transfer times between the Accumulator and Recycler were reduced significantly over the year and now average about 12-15 minutes with 90\% transfer efficiency.

- Despite the difficulties in improving the stacking rate, the accelerator complex produced roughly 20\% more antiprotons/week in FY 2007 as compared to FY 2006. This is a result of the reduction in the Accumulator-to-Recycler stacking interruption due to transfers, and the accompanying ability to restrict the Accumulator stack size to $60 \times 10^{10}$, where the stacking rate remains high.

- Improved luminosity lifetime

The luminosity lifetime continues to show modest improvement with the adjusted collision helix.

Reliability in FY 2007 was good. For the year, the average hours of luminosity per scheduled week of operations was 109 - an 8\% improvement over FY 2006. This was achieved despite two major failures of cryogenic components in October which interrupted operations for a total of three weeks, out of 44 weeks scheduled.

The antiproton stacking rate is the primary remaining key to performance in the Tevatron Collider. The design luminosity goal for FY 2007 was based on a stacking rate of $30 \times 10^{10}$ /hour. As noted above, the complex achieved $21-22 \times 10^{10}$ /hour. Achievement of the goal requires: 1) delivery of $8 \times 10^{12}$ protons onto the antiproton production target every 2.2 seconds; and 2) maintaining a production efficiency per proton of $23 \times 10^{-6}$ in the Antiproton Source. By the end of FY 2007, operations were with $\sim 8.5 \times 10^{12}$ protons on target, at a 2.4 sec cycle time, and a production efficiency of about $16 \times 10^{-6}$. A task force pursuing stacking rate improvements has measured the production efficiency through the Debuncher to be in the range $22-23 \times 10^{-6}$, and has concluded that the primary stacking bottleneck is in the stack-tail system in the Accumulator. A number of studies were completed and a new model of the system was constructed that explains the observations. On the basis of the model, a three-step plan was initiated: increasing the bandwidth (through the integration of equalizers), modifying the gain slope (through pickup reconfiguration), and improving mixing (through modification of the Accumulator lattice). Step 3 was completed, but steps 1 and 2 have encountered difficulties and remain in progress. Study time aimed at stacking rate improvements will be a continuing feature of operations in FY 2008. 
Luminosities in the range $22-28 \times 10^{31}$ are sustainable with a $21 \times 10^{10}$ /hour stacking rate as long as stores remain in the Tevatron for $\sim 24-30$ hours. For the balance of Run II, luminosity will be roughly proportional to the stacking rate.

\section{Status Relative to the FY 2007 Plan}

Table 1 shows planned and actual performance for the year.

Table 1. 2007 Tevatron Collider planned and actual performance.

\begin{tabular}{|l|l|l|l|}
\hline & Base Profile & $\underline{\text { Design Profile }}$ & Actual \\
\hline Median initial luminosity $\left(\mathrm{cm}^{-2} \mathrm{sec}^{-1}\right)$ & $10.7 \times 10^{31}$ & $31.9 \times 10^{31}$ & $19.6 \times 10^{31}$ \\
\hline Protons/bunch & $250 \times 10^{9}$ & $260 \times 10^{9}$ & $262 \times 10^{9}$ \\
\hline Pbars/bunch & $38 \times 10^{9}$ & $100 \times 10^{9}$ & $62 \times 10^{9}$ \\
\hline Effective emittance $(\pi \mathrm{mm}-\mathrm{mr})$ & 16 & 15 & 17 \\
\hline Beta at the IP $(\mathrm{cm})$ & 30 & 30 & 28 \\
\hline Hourglass factor & 0.65 & 0.65 & 0.67 \\
\hline Average stacking rate $\left(10^{10} /\right.$ hour) & 16 & 30 & 19 \\
\hline FY 2007 integrated luminosity (pb $\left.{ }^{-1}\right)$ & 762.7 & 1771.2 & 1311.3 \\
\hline FY 2007 integrated store time (hours) & 4444 & 4620 & 4777 \\
\hline FY 2007 scheduled uptime (hours) & & & 7088 \\
\hline FY 2007 unscheduled downtime (hours) & & & 1190 \\
\hline $\begin{array}{l}\text { FY 2007 unscheduled downtime as percent } \\
\text { of scheduled uptime }\end{array}$ & & & $17 \%$ \\
\hline
\end{tabular}

"Base" and "Design" correspond to end-of-year goals. Except for the last four rows, "Actual" corresponds to simultaneous performance on the median store of the last four weeks of operations.

\section{$\underline{\text { FY } 2007 \text { Neutrino Operations }}$}

A total of $1.9 \times 10^{20}$ protons were delivered to the NuMI target in FY 2007 - a $90 \%$ increase over the delivered protons in FY 2006, and roughly 87\% of the design goal for the year. Reliability was very good, with no extended interruptions to scheduled operations. The primary shortfall relative to the design goal arose from the cycle time - a 2.4-second Main Injector cycle was maintained through the year to accommodate the needs of the antiproton source, whereas the design goal assumed 2.0 seconds. Total protons delivered to NuMI to date stood at $3.6 \times 10^{20}$ at the end of the fiscal year.

Efforts continued on mitigation of tritium production and releases from the NuMI facility. There were no measurable concentrations of tritium in any of the streams flowing off the Fermilab site in FY 2007. A target chase dehumidification system was designed and is being installed in the FY 2007 shutdown. This system could lead to an additional factor of two reduction in tritium pumped from NuMI into the surface holding tank.

Booster Neutrino Beam operations were close to the design curve despite a 3.5-month interruption to implement repairs on failed intermediate absorber supports. Due to hydrogen embrittlement, the chains supporting two of the absorbers broke. During those periods when MiniBooNE was operating, performance was significantly above the design curve. MiniBooNE 
benefited from improved Booster performance, which allowed a significant number of protons to be delivered in parallel with antiproton production and NuMI running. A total of $1.5 \times 10^{20}$ protons were delivered to the Booster Neutrino Beam target during FY 2007, bringing total delivered protons to $9.5 \times 10^{20}$ by the end of the fiscal year.

\section{Shutdown Activites and Status}

At the end of FY 2007, eight weeks of the 2007 accelerator shutdown had been completed. The shutdown was scheduled for 12 weeks, including two weeks of Tevatron startup activities. Re-initiation of luminosity operations was scheduled for October 28. Nearly all activities were either completed or on schedule at the end of the fiscal year.

Summarized below in Table 2 is the status of major activities undertaken in the shutdown that were aimed at improved performance. There were myriad other activities that cannot be summarized here. Unless otherwise stated all listed activities were on schedule as of September 30, 2007.

Table 2. Status of major shutdown activities.

\begin{tabular}{|c|c|c|}
\hline Machine/Activity & Goal & Status \\
\hline \multicolumn{3}{|l|}{ Linac } \\
\hline LLRF upgrade & $\begin{array}{l}\text { Improved flexibility and reliability. } \\
\text { A Proton Plan activity. }\end{array}$ & $\begin{array}{l}\text { All supporting hardware was installed, but } \\
\text { difficulties with the processor board will } \\
\text { delay implementation until after the } \\
\text { shutdown. }\end{array}$ \\
\hline MTA $400 \mathrm{MeV}$ beamline & $\begin{array}{l}\text { Installation of components to allow } \\
\text { delivery of } 400 \mathrm{MeV} \text { beam to the } \\
\text { MuCool Test Area. }\end{array}$ & $\begin{array}{l}\text { Complete. All components scheduled for } \\
\text { installation during the shutdown were in. } \\
\text { Delivery of beam will require the further } \\
\text { installation of a "C-magnet", to be available } \\
\text { in late fall. }\end{array}$ \\
\hline \multicolumn{3}{|l|}{ Booster } \\
\hline New Booster correctors & $\begin{array}{l}\text { Loss reduction during acceleration. } \\
\text { A Proton Plan activity. }\end{array}$ & $\begin{array}{l}\text { Complete. Twelve correctors were installed } \\
\text { and will be operational during the coming } \\
\text { year. The remaining } 36 \text { will be installed in } \\
\text { the } 2008 \text { shutdown. }\end{array}$ \\
\hline \multicolumn{3}{|l|}{ Antiproton Source } \\
\hline $\begin{array}{l}\text { Replace leaking large quadrupole in the } \\
\text { Accumulator }\end{array}$ & Reliability risk reduction & Complete \\
\hline \begin{tabular}{|l|} 
Replace lithium lens \\
\end{tabular} & Increased antiproton yield & Complete \\
\hline \multicolumn{3}{|l|}{ Main Injector } \\
\hline Install MI-30 collimation system & $\begin{array}{l}\text { Increased intensity to NuMI: } \\
\text { control of losses to allow } \\
\text { implementation of multi-batch slip } \\
\text { stacking. A Proton Plan activity. }\end{array}$ & Complete \\
\hline \multicolumn{3}{|l|}{ Recycler } \\
\hline Install new DCCT & Improved diagnostics & Complete \\
\hline $\begin{array}{l}\text { Find/repair leaks in the antiproton } \\
\text { extraction line }\end{array}$ & Improved reliability & $\begin{array}{l}\text { Complete. Two leaks which had been } \\
\text { causing operational problems were } \\
\text { identified and repaired. }\end{array}$ \\
\hline \multicolumn{3}{|l|}{\begin{tabular}{|l|} 
Tevatron \\
\end{tabular}} \\
\hline Unroll Tevatron dipoles & Global coupling reduction & $\begin{array}{l}\text { Complete. All magnets were aligned to } \\
\text { within } 1 \mathrm{mr} \text { roll. ( } 55 \text { magnets unrolled) }\end{array}$ \\
\hline
\end{tabular}




\begin{tabular}{|l|l|l|}
\hline Machine/Activity & Goal & Status \\
\hline \hline Repair vacuum & Improved reliability & $\begin{array}{l}\text { Underway. Six (of 24) houses were warmed } \\
\text { up. Multiple leaks were identified and } \\
\text { repaired. One dipole was replaced in A1. } \\
\text { Two dipoles and one spool were replaced in } \\
\text { E1. }\end{array}$ \\
\hline & & \\
\hline NuMI & & \\
\hline Repair horn 2 water leak & & Complete \\
\hline Fill decay pipe with He & Operability & Underway \\
\hline Add target chase dehumidification & Safety & Underway \\
\hline & Tritium reduction & \\
\hline Infrastructure Maintenance & & \\
\hline Replace 345 KV power poles & & Complete \\
\hline
\end{tabular}

E-830 / Collider Detector at Fermilab (CDF) (M. Convery, P. Schlabach, P. Wilson)

During FY 2007 a total integrated luminosity of $1.3 \mathrm{fb}^{-1}$ was delivered to CDF, of which $1.1 \mathrm{fb}^{-1}$ was recorded to tape. At the end of FY 2007, a total of $3.2 \mathrm{fb}^{-1}$ had been delivered to the experiment in Run II (Figure 2). The CDF detector operated with a total data collection efficiency of $83 \%$ including dead time associated with trigger acceptance, operational inefficiencies (e.g. starting and stopping runs) and downtime from detector problems.

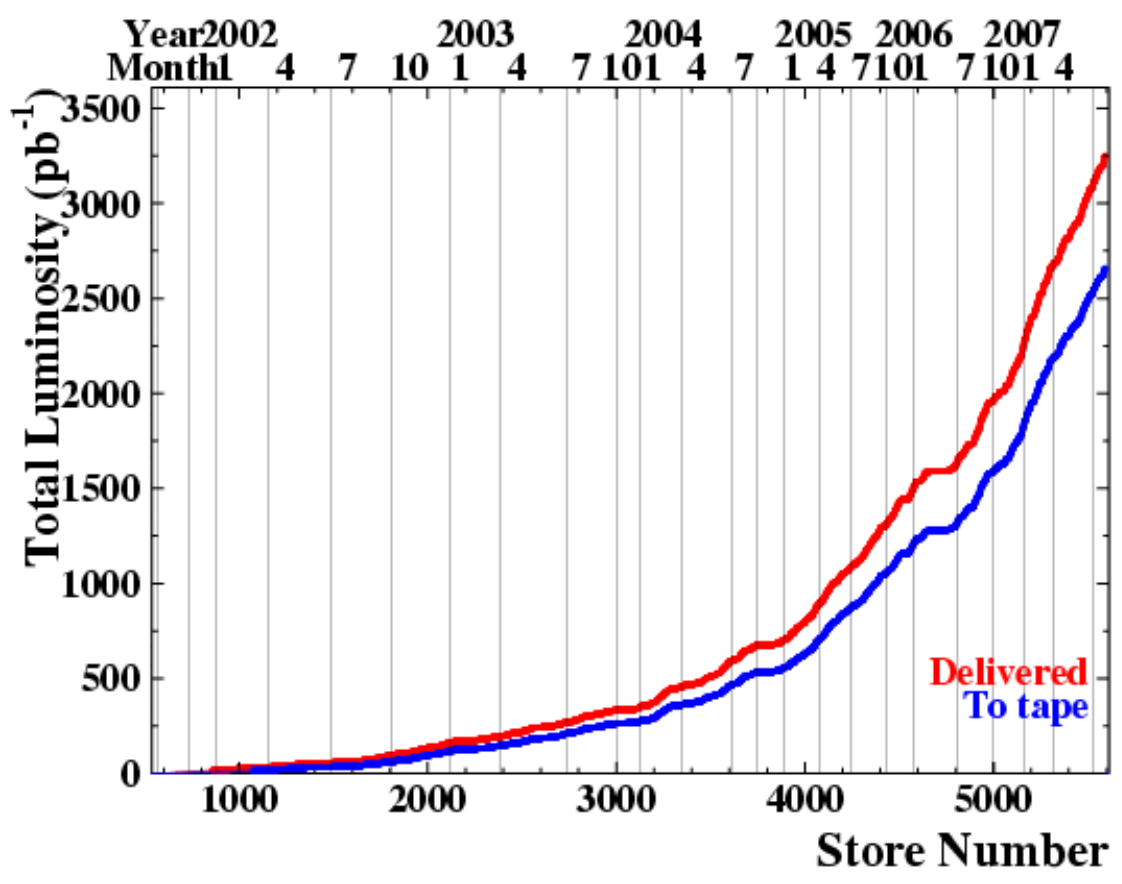

Figure 2. Delivered and recorded luminosity at CDF in fiscal years 2001-2007.

The Tevatron delivered record instantaneous luminosities reaching close to $3 \times 10^{32} \mathrm{~cm}^{-2} \mathrm{sec}^{-1}$ with many stores starting above $2.5 \times 10^{32} \mathrm{~cm}^{-2} \mathrm{sec}^{-1}$. The final bandwidth improvements of the Run IIb upgrades were commissioned early in FY 2007, allowing CDF to 
operate with good livetime without throttling back on high-priority physics triggers. All the CDF detector systems handled the high instantaneous luminosities without significant problems and as projected by previous studies.

Commissioning of the stereo upgrade to the Level 1 Track Trigger was completed in October 2006. This upgrade was needed for running at instantaneous luminosities in excess of $2 \times 10^{32} \mathrm{~cm}^{-2} \mathrm{sec}^{-1}$. The upgrade hardware finds track segments in stereo layers of the central tracker and associates them with axial tracks from the existing Level 1 track trigger processor to reduce the rate of fake tracks. The stereo track segments are used to confirm that the axial track has corresponding segments in the stereo layers, significantly reducing the rate of fake tracks. This confirmation is available for use in both the Level 1 and Level 2 trigger decisions. The upgraded system was immediately put to use in the critical Level 2 high $\mathrm{P}_{\mathrm{t}}$ muon triggers used for top, Higgs, and other searches. By March of 2007, the stereo information was implemented in all planned areas of the physics program, which significantly increased the instantaneous luminosity at which all B physics signatures could be triggered efficiently.

A new data-logging system was commissioned on schedule in November 2006. The system takes events from the Level 3 farms, buffers them onto local disks, and then puts them onto tape. The old system had hardware and software components that were obsolete and without maintenance support. The new data logger has a bandwidth in excess of $80 \mathrm{MB} / \mathrm{sec}$, and will not be a limitation to sustained data-taking, even at instantaneous luminosities in excess of $3.0 \times 10^{32} \mathrm{~cm}^{-2} \mathrm{sec}^{-1}$.

These upgrades, along with others commissioned in FY 2004-05, ensure that the CDF detector will continue to run well at the highest instantaneous luminosities the Tevatron delivers. To take full advantage of the delivered luminosity, development of the trigger systems and trigger table continued. In FY 2007 the Level 2 calorimeter cluster finder, which is used for electron, photon, jet, and tau triggers, was improved by taking advantage of the Pulsar boards which were designed for the Run IIb Level 2 trigger upgrade. This improvement moves the clustering algorithm from highly restrictive firmware to software in the trigger decision computer. This permits the use of an algorithm very similar to that used in the Level 3 trigger and offline. Commissioning of the improved cluster finder was completed immediately prior to the 2007 shutdown and all electron, photon, jet, tau, and missing transverse energy triggers have been updated for data taking in FY 2008.

One significant detector problem arose during the year. In the spring of 2007, a leak developed in the cooling system for the silicon vertex detector. The leak was in a cooling circuit for electronic circuits used to read out the inner most layer (L00) and outermost layers (ISL) on the east half of the detector. These circuits are located close to the silicon sensors inside the inner bore of the drift chamber, about 0.5 meter from the chamber face, preventing access to cooling tubes from the outside. The source of the problem was isolated from inside using borescopes and medical catheters. From the point that the leak developed in mid-May until the shutdown in August, the east half of L00 and ISL were powered off. The primary five-layer SVX detector was not affected by the leak and remained in use, as did the Silicon Vertex Trigger which only uses the SVX layers. The loss of half of L00 reduces the effectiveness of b-tagging, but the data is still useful for all physics analyses. Approximately $270 \mathrm{pb}^{-1}$ of data were collected with the reduced silicon detector capability. 
Immediately after the leak developed, a task force was created to determine the cause of the leak, develop a repair technique, and address possible long-term implications. The cause was determined to be corrosion around welded joints in the aluminum cooling tubes induced by acidic coolant. Analysis of the coolant indicated that the ethylene glycol in the coolant partially degraded, producing formic acid. The acidity of the coolant was already being addressed when the leak developed and has remained under control. The corrosion is limited to the areas around the aluminum welds which were only utilized in the cooling for the L00/ISL electronic circuits. All other aluminum cooling components are joined with epoxy. A repair technique was developed using epoxy deposited on the inside of the tubing.

Repair of the L00/ISL cooling was the primary focus at CDF for the 2007 shutdown. The inner layers of the silicon detectors had received sufficient radiation dose by the beginning of the shutdown that they had to be kept cold $\left(<0^{\circ} \mathrm{C}\right)$ to prevent significant degradation of performance. An ultra-dry work environment was developed to permit access to the silicon detectors without risk of condensation on the very sensitive cold components. The leaking joints on the east cooling circuit were repaired, and similar damage was observed on the west side and repaired. Other cooling lines with epoxy joints were examined. No corrosion was observed in these other lines.

During the 2007 shutdown there were also routine repairs and maintenance to all CDF detector components. Preventive maintenance was performed on detector infrastructure. For example, all of the power supplies for the calorimeter readout crates were overhauled to address a few failures indicating components approaching end-of-life. A new gas filtration system was completed for the central drift chamber (COT). This system was designed in response to gain loss which was observed earlier in Run II. The aging had been reversed by adding a small amount of oxygen to the argon-ethane mix. The new system has the capacity to filter gas that is recirculated back into the COT. At the end of the 2007 shutdown, commissioning of this system was near completion.

The shutdown also provided an opportunity for many people to tour the CDF collision hall. In all, nearly 200 people took advantage of the chance to see the detector. There were visitors from domestic and foreign funding agencies, Congress, conferences and workshops, other experiments and laboratories, members of the press, and several film crews. Fermilab employees from almost every division and of many different job classifications also toured, as did many incoming CDF students and post docs.

In addition to the improvements in trigger and detector systems, there were also improvements in shift operations. The changes were devised to reduce the personnel requirements for shift operations. They streamlined operations while increasing data-taking efficiency. The number of physicists on each eight-hour shift was reduced from four to three. This change was made possible by automating many routine tasks and re-arranging the control room to make the remaining crew members more effective. In addition, CDF instituted shift monitoring from a remote control room by implementing web-based monitoring tools and utilitizing video-conferencing communications between the shift crews at the two sites. Approximately one week per month, the owl shift data quality monitoring was done remotely from Pisa, Italy. This system allows foreign collaborators to participate in operation of the experiment much more easily. Just prior to the 2007 shutdown, the system was tested with remote shifts from Japan. Other institutions are interested in participating as well. These 
changes to the shift operations were made without a loss in data-taking efficiency (Figure 3). In fact, many of the changes made to facilitate the remote control room have made it easier for detector experts to monitor data-taking and data quality from off-site. This reduces response time in case of problems, and increases the probability that problems are spotted.

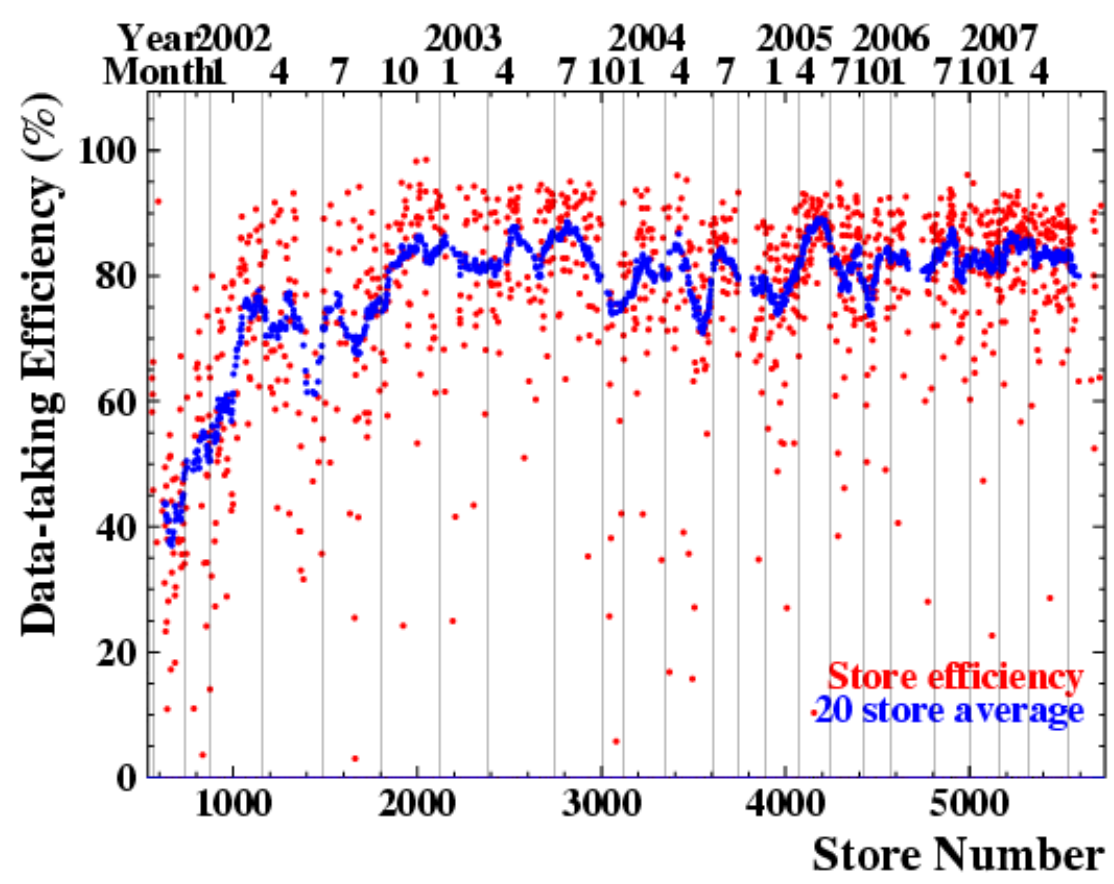

Figure 3. Data-taking efficiency for CDF in fiscal years 2001-2007.

As post-shutdown startup activities commenced, the detector, trigger, readout, and infrastructure were in good shape. Important startup tasks included training shift crews for datataking with beam, final commissioning of the L2 stereo trigger upgrade, and implementation of trigger paths that take advantage of the L2 calorimeter trigger upgrade.

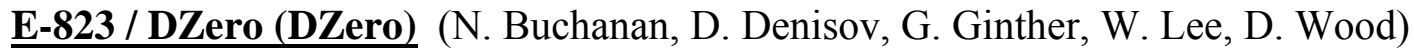

Fiscal year 2007 began with the accelerator complex in routine operation, and ended during a long shutdown. The accelerator complex delivered an integrated luminosity of $1.32 \mathrm{fb}^{-1}$ to DZero during FY 2007. DZero recorded $1.15 \mathrm{fb}^{-1}$, corresponding to an operating efficiency of $87.1 \%$ during that time period. Figure 4 illustrates the detector efficiency as a function of time. This represents the best fiscal year operating efficiency achieved by DZero during Run II, and the data collected in FY 2007 more than doubled the integrated luminosity recorded during any previous fiscal year. These achievements were made possible by increases in instantaneous and integrated luminosities delivered by the accelerator complex, as well as due to detector improvements, several of which were introduced during the 2006 long shutdown. These improvements, including an additional layer of silicon just outside the beam pipe, and new 
Level 1 track and calorimeter triggers, performed very well during this fiscal year, and resulted in substantial enhancements in the available data.

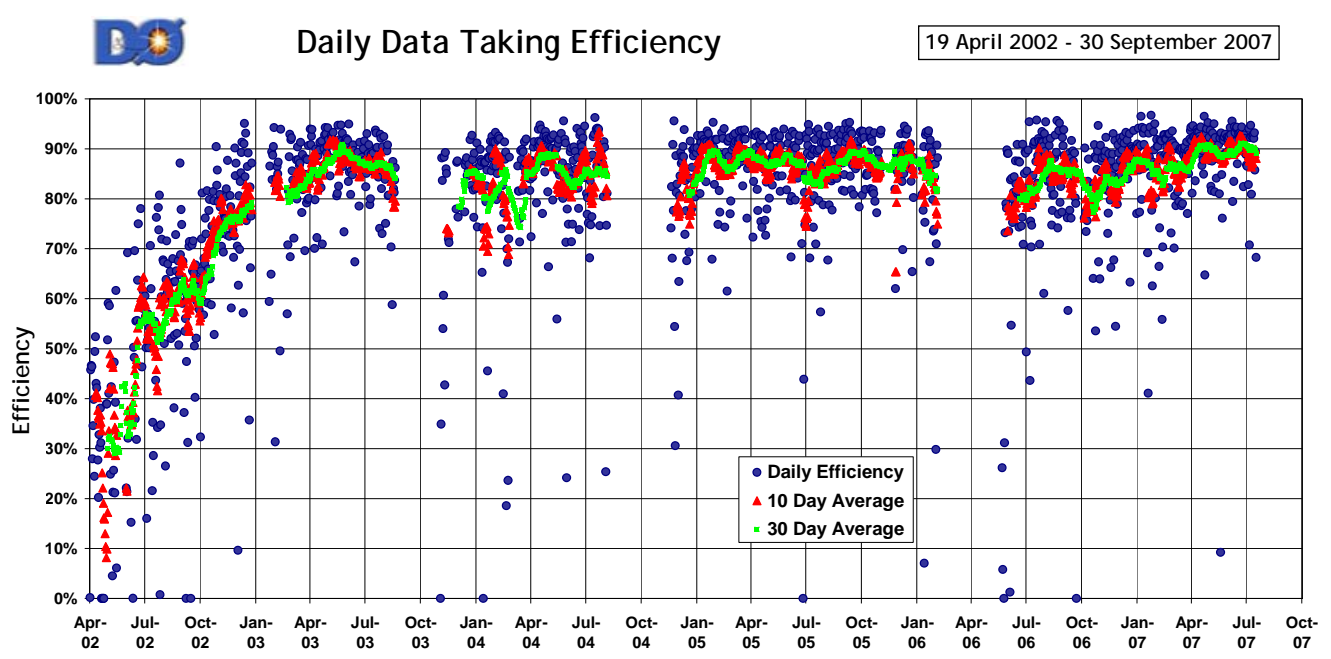

Figure 4. DZero data-taking efficiency as a function of time during Run II. The blue dots represent a daily average, the red triangles represent a 10-day average, and the green squares represent a 30-day average.

While the accelerator increased peak instantaneous luminosities, DZero made improvements to the trigger list to take advantage of the trigger improvements introduced during the 2006 long shutdown. In parallel with efficient data acquisition, developments to accommodate matching of calorimeter and track objects at the lowest-level trigger stage continued, improving trigger rejection rates and efficiencies. To accommodate this Level 1 calorimeter-track-match trigger, the latency for all Level 1 trigger decisions had to be delayed by $396 \mathrm{nsec}$. This delay was introduced in December of 2006. The Level 1 calorimeter-trackmatch trigger was incorporated into the standard trigger lists soon thereafter. In March of 2007, an additional 120 nodes were added to the Level 3 trigger farm to enhance performance and reliability. The software that implements the Level 3 tracking was also significantly enhanced during this fiscal year. Peak luminosities increased rapidly after the 2006 long shutdown, with the current DZero record at $296 \times 10^{30} \mathrm{~cm}^{-2} \mathrm{sec}^{-1}$. The DZero detector was able to handle this record peak luminosity without prescaling the core physics program triggers.

DZero continues efforts to streamline operations in the face of increasing instantaneous luminosity, and is refining its operations in an effort to improve efficiency. As of June, the responsibilities for round-the-clock monitoring of the Silicon Microstrip Tracker and the Central Fiber Tracker (and the associated trigger systems) were combined to reduce the number of control room shifters.

The Central Fiber Tracker (CFT) performed well, and physics analyses are making good use of the enhanced tracking capabilities of the Run II tracking detectors. However, the performance of the Analog Front End boards (AFE) used to instrument the Visible Light Photon Counter readout of the CFT was expected to degrade as the instantaneous luminosity increases. Due to the high occupancies that are encountered in the CFT channels, the readout electronics can suffer saturation, resulting in reduced efficiency. In addition, the original electronics 
exhibits undesirable channel-to-channel and crossing-to-crossing pedestal dependence, effectively forcing an unfortunate choice between reducing performance and increasing noise occupancy and readout deadtime. The AFEII readout board was developed to address these features. The AFEII is based upon a new readout chip that was designed specifically for this purpose. Production of the AFEII boards was completed during September 2006. Installation of the AFEII boards took advantage of access opportunities during brief Tevatron downtimes, and the entire Central Fiber Tracker and Central Preshower detectors were fully instrumented with AFEII boards prior to the 2007 long shutdown. Consequently, thresholds employed in track reconstruction have been re-evaluated, resulting in improved track reconstruction efficiency. The AFEII boards also provided the ability to operate the Central Preshower channels with reduced gain, resulting in extended dynamic range for electron and photon detection. The AFEII readout chip provides the additional capability to measure the time of arrival of the signals from the CFT. These timing measurements could be used to suppress accidental clusters during track reconstruction, and tests of this capability were performed prior to the 2007 long shutdown.

The 2007 long shutdown began on August 5, and DZero used the time to perform maintenance and introduce improvements. The luminosity monitors were replaced to minimize the impact of radiation damage to the scintillators. A substantial effort was undertaken to study, and where possible, recover failed Silicon Microstrip Tracker readout channels. Firmware and software were developed to utilize the timing readout feature of the AFEII boards for the CFT. The readout for the Forward Preshower detector was instrumented with AFEII boards. Significant effort was invested in enhancing the performance of the Intercryostat Detector. Routine and preventive maintenance, as well as single-channel recoveries were also performed on the various sub-detectors. For example, a small leak which had developed in the toroid water cooling system was repaired.

During the 60 weeks of operation since the end of the 2006 long shutdown, DZero recorded a total of $1.45 \mathrm{fb}^{-1}$ of the $1.68 \mathrm{fb}^{-1}$ of delivered integrated luminosity, which corresponds to an average efficiency of $86.4 \%$, and the total recorded Run II data exposure more than doubled from $1.35 \mathrm{fb}^{-1}$ to $2.80 \mathrm{fb}^{-1}$ (as illustrated in Figure 5). The detector ran smoothly and efficiently, and is being prepared to effectively handle larger instantaneous and integrated luminosities anticipated for the near future. 


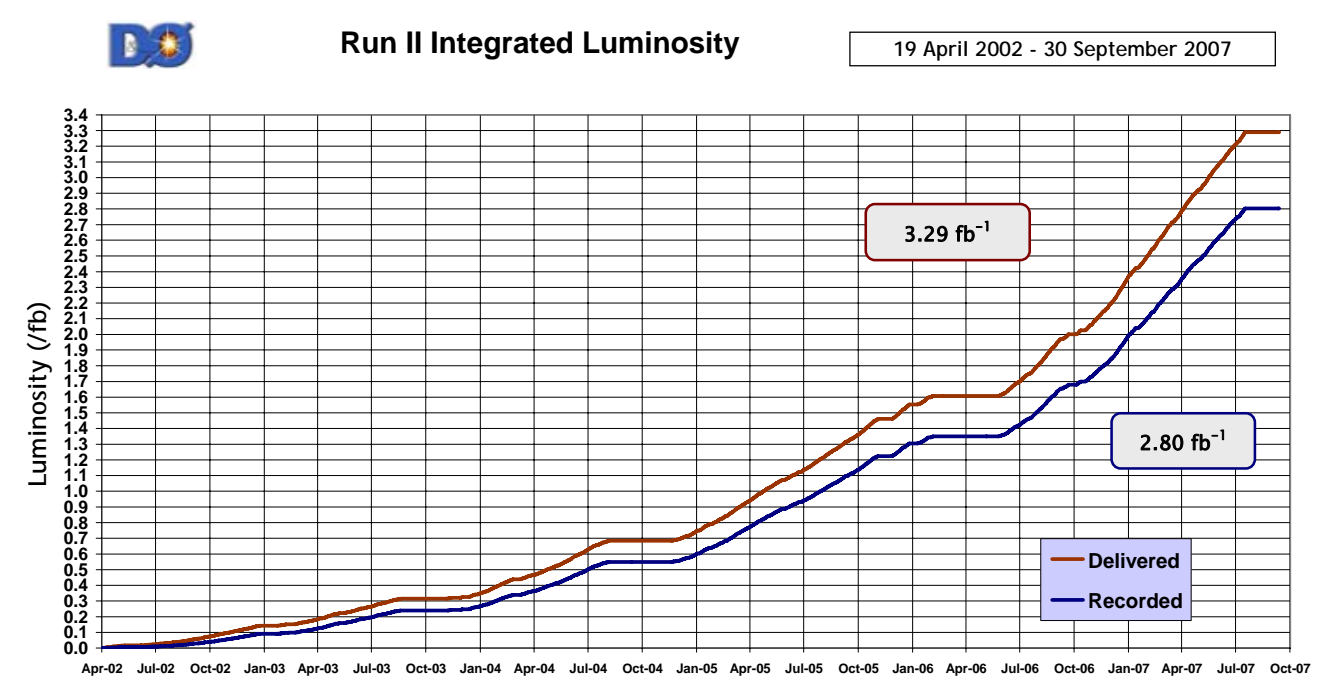

Figure 5. Delivered (red line) and recorded (blue line) luminosity at DZero as a function of time during Run II.

NuMI / E-875 Main Injector Neutrino Oscillation Search (MINOS) (A. Habig, R. Plunkett)

$\underline{\mathrm{NuMI}}$

The NuMI beam facility operated reliably and well during FY 2007, delivering $1.9 \times 10^{20}$ protons to the NuMI target between October 1, 2006 and July 15, 2007. This exceeded the goal of $1.5 \times 10^{20}$ FY 2007 protons. Figure 6 shows the protons delivered per week and the integrated total during FY 2007. While there were no extended downtimes this year, there were several downtimes of a few days, causing the weekly exposure to fall below the normal running conditions of $5 \times 10^{18}$ protons, and the NuMI beam stopped for shutdown earlier than the rest of the accelerator complex to prevent the effects of a cooling water leak repair in focusing horn $\# 2$ from confusing a necessary planned study of the beam-off decay of tritium emitted in the NuMI target chase. The few operational problems are detailed below, but the overall performance of the beam components was exceptional. The target (the second one used in NuMI) has survived $1.5 \times 10^{20}$ protons arriving at a typical power of $175 \mathrm{~kW}$, while both horns are original and have exceeded their design goal of ten million pulses, experiencing $1.7 \mathrm{GW}$-hours of beam. A spare target and a set of spare horns are now ready to be installed should components fail in the harsh environment during future operations. 


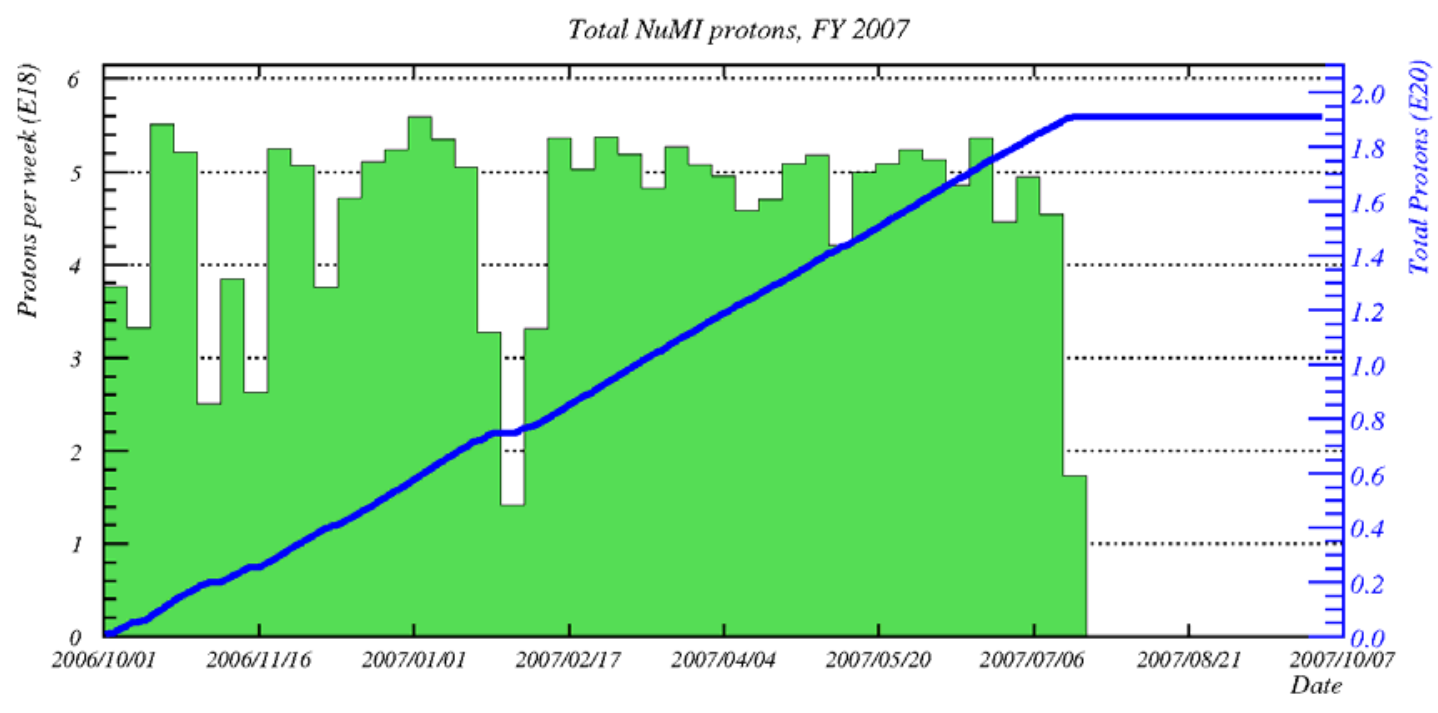

Figure 6. NuMI's weekly total protons on target (histogram, left axis) and integrated FY 2007 total proton exposure (line, right axis). A total of $1.9 \times 10^{20}$ protons were delivered to the NuMI target in FY 2007. The scheduled 2007 long shutdown is the empty time on the right of the x-axis.

Improvements made to the NuMI beam this year were dominated by the successful slipstacking of high-intensity proton bunches in the Main Injector and their extraction onto the NuMI target. In addition, preparations and tests of a 2.2 second cycle time were made. Elevenbunch slip-stacking was performed, and tests of intensities on the NuMI target of up to $4 \times 10^{13}$ protons per pulse $(300 \mathrm{~kW})$ were successful.

Three problems caused multi-day NuMI downtimes during this period. The first was a failure of the NuMI kicker charging power supply and subsequent intermittent interlock in early November 2006. Later in November the target hall chiller unit compressor was replaced. The mid-December dip was due to accelerator problems upstream, but the two bad weeks at the end of January 2008 were necessitated by the replacement of a leaking magnet in the NuMI extraction line from the Main Injector. In all cases repairs were completed rapidly and the beam was back online in short order. The cooling leak in horn \#2 was very similar to one of those repaired in the previous year. It was caused by a design flaw at the ceramic junction between the water line and the horn itself, and has been corrected in the spare horns.

Mitigation of tritiated humid air entering the NuMI tunnel passageway, and thereby the sump water, was successful. It maintained an improvement of approximately $50 \%$ over previous tritium levels. This mitigation consisted of aggressive dehumidification of the air entering the tunnel from the target hall.

The shutdown work which occupied the remainder of the fiscal year was used not only to repair the aforementioned horn \#2 water leak, but also for two major projects. The first was to install a large dehumidification system in the NuMI target hall for further tritium mitigation via dehumidification of the target chase air itself. The second was to convert the NuMI decay pipe from vacuum to a helium atmosphere in order to eliminate mechanical stresses on the upstream NuMI decay pipe window. 
The stability of the beam over time and the understanding of the resulting neutrino spectrum has been very good. No change in neutrino spectrum has occurred over time for a given physical arrangement of the target and horns. Changing the relative positions of these components produces a different neutrino spectrum, a feature which has allowed the careful testing of the beam modeling process. As a result, a misalignment of the target by one centimeter longitudinally was noticed from the MINOS data alone, as shown in Figure 7. The misalignment was later confirmed by direct survey.

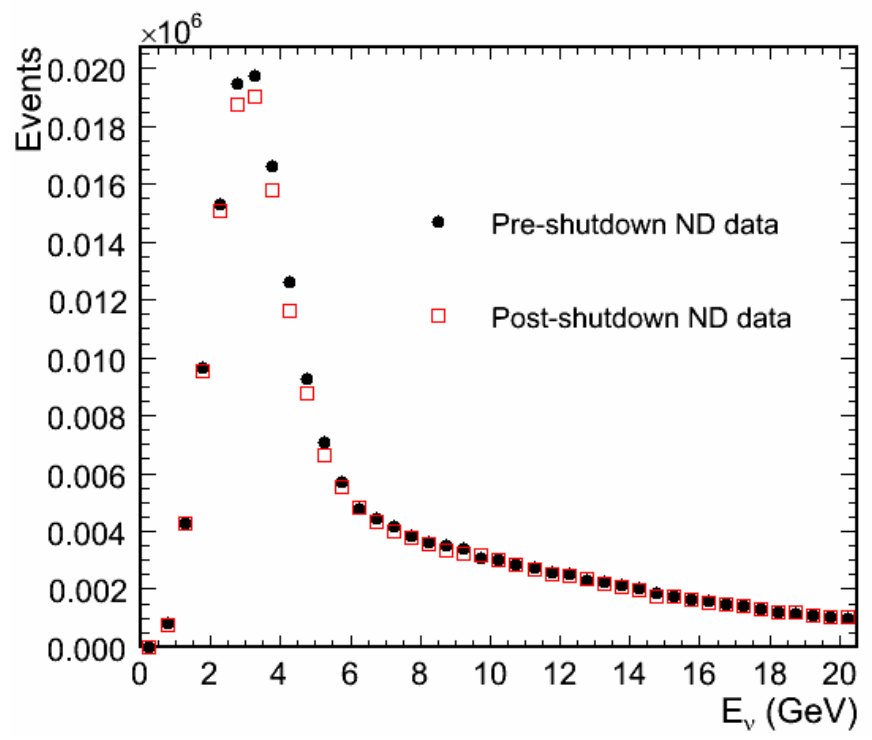

Figure 7. The reconstructed neutrino energy spectrum in the MINOS Near Detector (ND), comparing pre- and post- 2006 shutdown data. The differences in the $5 \mathrm{GeV}$ region were compared to beam Monte Carlo predictions, suggesting a $1 \mathrm{~cm}$ target misalignment which was later confirmed by direct survey.

\section{MINOS}

The MINOS experiment continued its efficient operation in FY 2007. Both Near and Far Detectors operated at extremely high efficiencies when the beam was on, with the statisticslimited Far Detector taking good data more than $98 \%$ of the time during beam and $96 \%$ of the time overall (including scheduled shutdown maintenance). The typical state of the Far Detector is zero dead channels, with one problem developing every few weeks and being fixed within a day, so no changes have had to be made there. The Near Detector electronics underwent a complete retrofit during the shutdown to replace unreliable fuses on the front end boards, which had resulted in several channels per week needing to be repaired. This swap seems to have eliminated this failure mode, and it is expected that this will result in the two detectors both having no holes most of the time.

The shutdown time was also used to take some reversed-magnetic-field cosmic-ray data at the Near Detector. This will allow the reduction of systematic errors in the cosmic-ray muon charge-ratio measurement at the Near Detector, to better compare to the ground-breaking highenergy/charge-ratio measurement at the Far Detector. The Far Detector measurement [PRD 76, 052003 (2007)] is the first measurement of associated kaon production around $8 \mathrm{TeV}$. The 
shallower Near Detector sees muons from cosmic-ray primaries of several hundred $\mathrm{GeV}$, an energy range dominated by pion production, and directly comparable to the extensive chargeratio measurements done by the $\mathrm{L} 3+\mathrm{C}$ experiment.

The initial MINOS neutrino oscillation results were based on data ending in February 2006, with $1.27 \times 10^{20}$ protons on target, and were published in FY 2007 [PRL 97, 171801 (2006)]. Data taken through April 2007 has now been analyzed, the reconstruction algorithms improved, and a neutrino oscillation analysis performed on the resulting $2.50 \times 10^{20}$ proton data set. The resulting doubling of data and improved analysis techniques have greatly improved our knowledge of the $v_{2} \leftrightarrow v_{3} \sin ^{2}\left(\theta_{23}\right)$ oscillation parameter, as shown in Figure 8 .

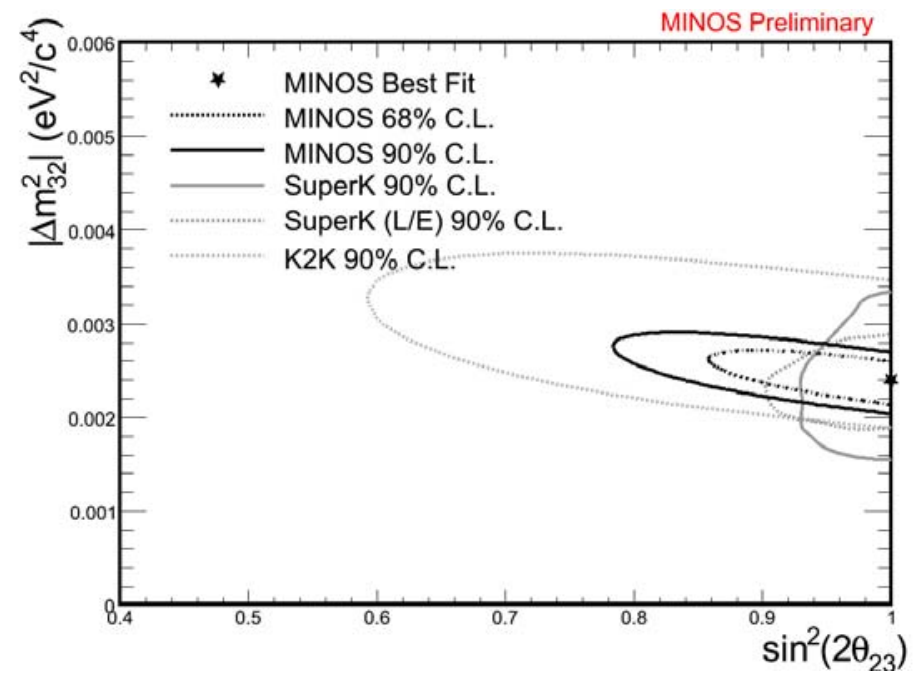

Figure 8. The allowed region in parameter space from the analysis of charged current $v_{\mu}$ interactions in MINOS resulting from $2.5 \times 10^{20} \mathrm{NuMI}$ protons on target, compared to previous experiments.

The Near Detector affords a very high-statistics sample of neutrino interactions. This allows the detailed study of neutral-current interactions, a topic where great uncertainty afflicts current measurements, and which is both an important background to many other neutrino measurements and a direct measurement of total neutrino flux irrespective of any oscillation phenomena. The first $1.2 \times 10^{20} \mathrm{NuMI}$ protons-on-target were used to generate Figure 9, a measurement of the spectrum of neutral-current interactions in the Near Detector. This is the first step needed for many upcoming analyses. 


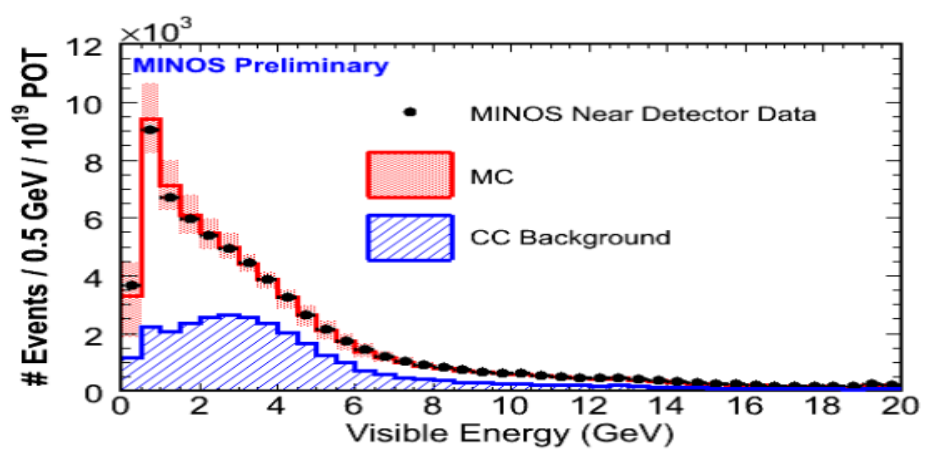

Figure 9. The neutral-current-selected energy spectrum in the Near Detector. Poorly reconstructed events are removed using a combination of timing and topology cuts. The black dots are the data, the red bars are the statistical and systematic error bars around the Monte Carlo simulation, and the shaded blue is the charged-current background. The neutral-current selection has purity of approximately $50 \%$. The data correspond to $1.22 \times 10^{20}$ protons on target.

\section{E-944 / MiniBooNE (S. Brice, R. Van de Water)}

Starting in early January 2006, a two-week shutdown of the Booster Neutrino Beam and MiniBooNE operations occurred to swap out the positive horn charging supplies and replace them with negative supplies. Work also was done to the power bus to make future change-outs easier and quicker, reducing a two-week job to less than one week. The power supply changeout was successful, and on January 18, 2006 the Booster Neutrino Beam began operations with a reversed-horn current of $-175 \mathrm{kA}$, focusing negative charged particles, which preferentially produce antineutrinos with a roughly $30 \%$ admixture of neutrinos.

Figure 10 shows the weekly performance of the Booster Neutrino Beam since the start of running in 2002. The antineutrino running is shown after the solid line. The first large gap is the 2006 long shutdown which started at the end of February, and lasted three months. The second gap is the Booster Neutrino Beam shutdown from January to April 2007 to repair a problem in the $25 \mathrm{~m}$ absorber region. The performance of the Booster in FY 2007 has been exceptional. During the last half of FY 2007 the weekly protons-on-target (POT) delivered to the Booster Neutrino Beam was over $5 \times 10^{19}$. What makes this number impressive is that this was while full beam was simultaneously delivered to the NuMI target. 


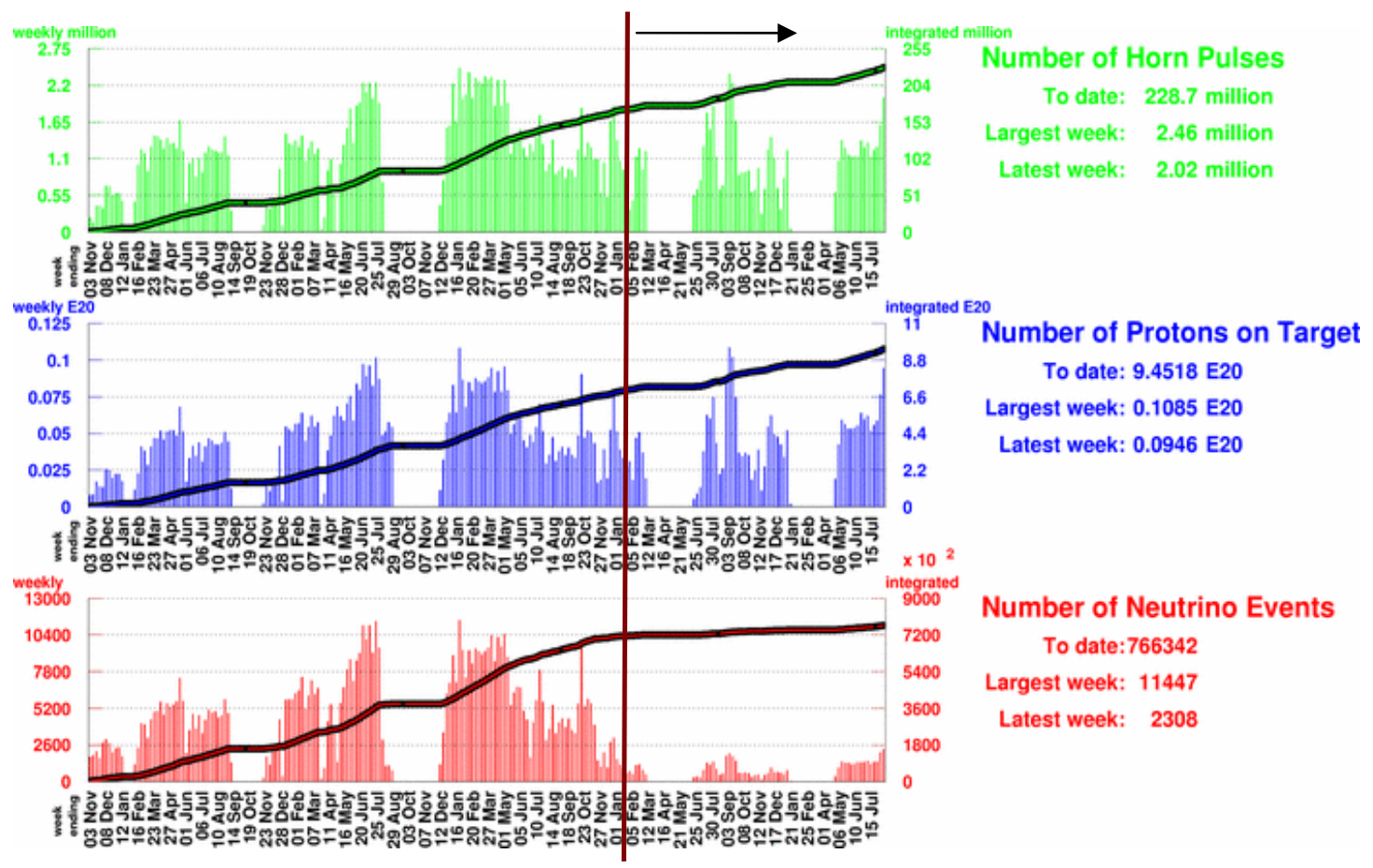

Figure 10. Weekly beam summary since the start of MiniBooNE operation in 2002. Antineutrino running is shown to the right of the solid vertical line.

In spite of the four-month repair shutdown, MiniBooNE collected a total of $2.33 \times 10^{20}$ POT in antineutrino mode in one and a half years (see Table 3 ). Even after neutrino rate corrections for the absorber plates, this meets the MiniBooNE physics goal of $2.0 \times 10^{20}$ POT. This milestone achievement was due to pushing the Booster to peak performance and repairing the $25 \mathrm{~m}$ absorber problem professionally and efficiently.

Table 3. Integrated protons-on-target in antineutrino mode for various run conditions. The third column includes correction for reduced neutrino rates due to the absorber failure.

\begin{tabular}{|l|c|c|}
\hline Condition & $\begin{array}{c}\text { Total } \\
\times 10^{20} \mathrm{POT}\end{array}$ & $\begin{array}{c}\text { Corrected } \\
\times 10^{20} \mathrm{POT}\end{array}$ \\
\hline \hline No absorber & 1.15 & 1.15 \\
\hline One absorber plate down & 0.57 & 0.45 \\
\hline Two absorber plates down & 0.61 & 0.43 \\
\hline Totals & 2.33 & 2.03 \\
\hline
\end{tabular}

The $25 \mathrm{~m}$ absorber problem was first noticed shortly after the beam came back up after the 2006 long shutdown. As can be seen from Figure 11, there is an initial 20\% drop after week 18 (June 2007), then a further drop to $30 \%$ around the $34^{\text {th }}$ week (August 2007). The problem only manifested itself as a drop in neutrino event rate, which is low to begin with in antineutrino mode. After detailed analysis ruled out various components of the experiment, e.g proton beamline, horn, target, detector, etc., it was realized that the problem must be in the decay pipe 
region. The decision was made to shut down the beamline and open up the decay region for inspection. After an initial investigation it was found that two out of eleven 25-ton steel absorber plates had fallen into the decay region.

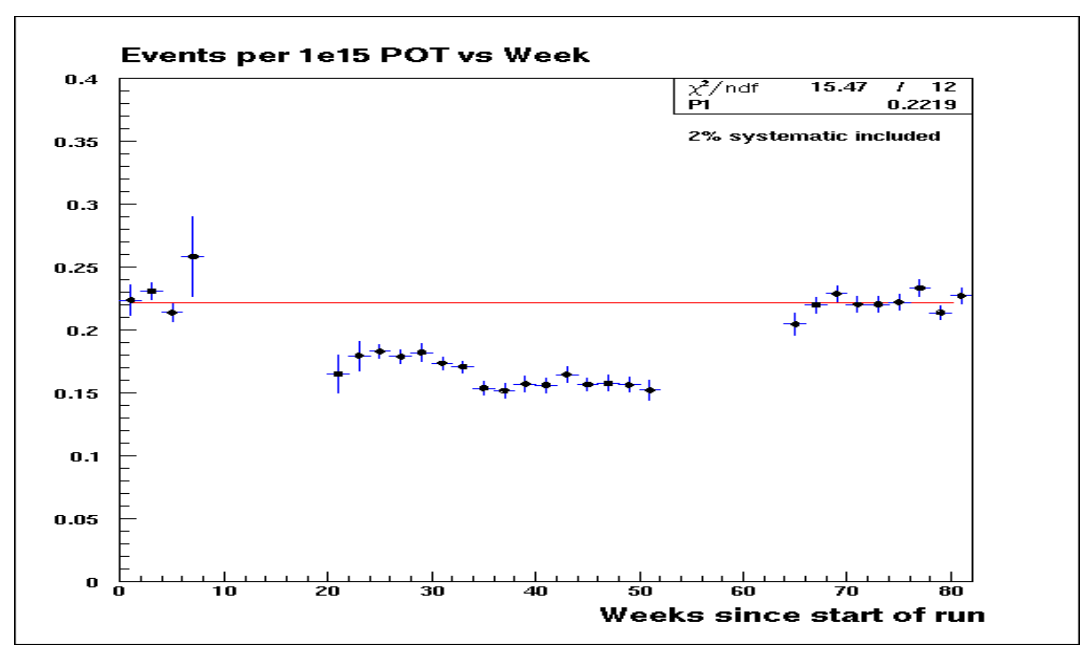

Figure 11. Neutrino events per POT for the antineutrino run starting in January 2006. The red line shows the expected value for normal running. After the 2006 long shutdown (first gap), the rates decrease due to the $25 \mathrm{~m}$ absorber problem. A repair was effected from week 52 to 64 . Since then, the neutrino event/POT values have returned to normal.

An Accelerator Division task force was formed to understand the cause of the problem and formulate a repair plan. In the highly corrosive environment of the decay region (due to radiation and dampness), the hardened steel chains that were supporting the 25-ton plates had become embrittled by hydrogen ingress. The subsequent cracking was accelerated by the constant load, which ultimately resulted in failure of the chains.

Once the cause of the chain failure was understood, a robust repair was formulated. The steel chains were replaced by stainless steel rods. The rod removed the saddle point weakness of the chains, and the stainless steel is known to be impervious to hydrogen embrittlement. The repair was quickly enacted, and the beamline brought back online, all in about four months. Once a year, an inspection of the decay region will be done to ensure that the 25 -ton plates are in no danger of falling again.

Subsequent analysis of the absorber problem data has shown it to be completely recoverable. Detailed modeling of the absorber effects are understood and the data can be corrected for use for neutrino oscillation and cross section analyses.

An important aspect of continued running of MiniBooNE is the reliability of the beamline, horn, and detector systems. For the FY 2007 antineutrino run period, the combined beamline and detector uptime was over $95 \%$ (this ignores the $25 \mathrm{~m}$ repair downtime). The most crucial element, the horn, has collected over 135 million pulses, surpassing the first horn, which failed two years ago with 94 million pulses (both these numbers are world records). This second horn continues to run flawlessly, and with no sign of problems. In the unfortunate event of 
failure, a spare, third horn and target are available. It would require three weeks to swap in the replacement system.

\section{E-954 / SciBooNE (T. Nakaya, M. Wascko)}

Civil construction for the SciBooNE Detector Hall began in September 2006, with a groundbreaking ceremony held on 20 September. Beneficial occupancy was gained in January 2007, and all electrical and other modifications were complete by April.

The SciBooNE vertex detectors are the Scintillating Bar (SciBar) and Electron Catcher (EC) detectors, which were shipped from Japan where they had been used in the K2K experiment. The assembly of SciBar and the EC was carried out in the CDF Assembly Pit. The EC components were tested and assembly was completed by November 2006. Cosmic data from the EC were collected with a standalone DAQ in December. The SciBar scintillator planes were installed in January 2007. All wavelength shifting fibers and multi-anode photomultipliers were installed into the SciBar planes in February. The first cosmic-ray data with the SciBooNE data acquisition (DAQ) system were recorded in March.

The Muon Range Detector (MRD) was constructed from recycled parts in Lab E/F in the Neutrino Area. Preparation of the steel planes was completed in January 2007. The scintillation counter installation was completed in March, when the MRD recorded its first cosmic-ray events.

The detectors were installed in the SciBooNE Detector Hall April 23-26. Cabling of the systems was completed and integration of all detector components into the DAQ system was completed in May. The first events from the neutrino beam were recorded May 29 and presented at the Neutrino Interactions Workshop (NuInt07) held at Fermilab. Figure 12 shows an event display of SciBooNE's first recorded neutrino interaction with energy deposited in all three subdetectors. Two weeks of commissioning followed, at the end of which all detector systems were performing well.

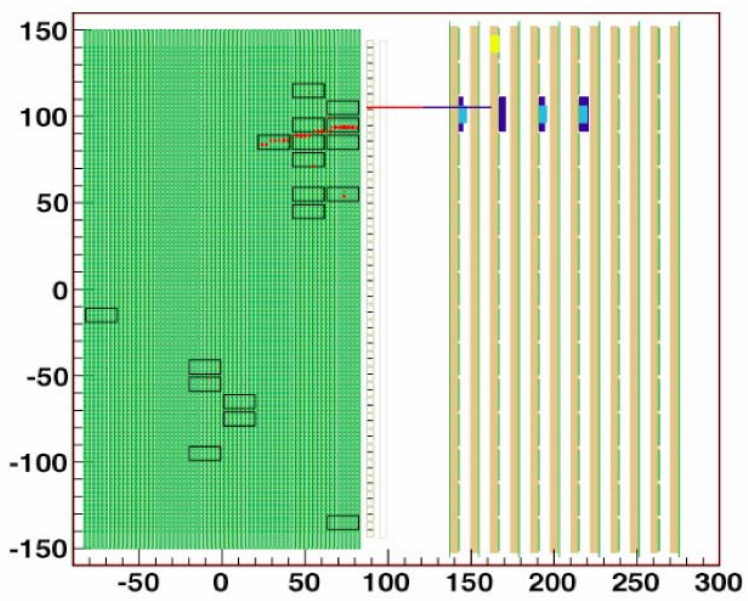

Figure 12. Event display of SciBooNE's first recorded beam event, with energy deposited in all three subdetectors. 
On June 12, SciBooNE's FY 2007 beam run began. The Booster Neutrino Beam horn was in reversed polarity mode, producing a beam comprised of approximately two-thirds antineutrinos and one-third neutrinos at the SciBooNE detector. The experiment was exposed to $5.45 \times 10^{19}$ protons on target (POT) before the accelerator shutdown on August 3. Figure 13 shows the accumulation of POT for SciBooNE's FY 2007 beam run.

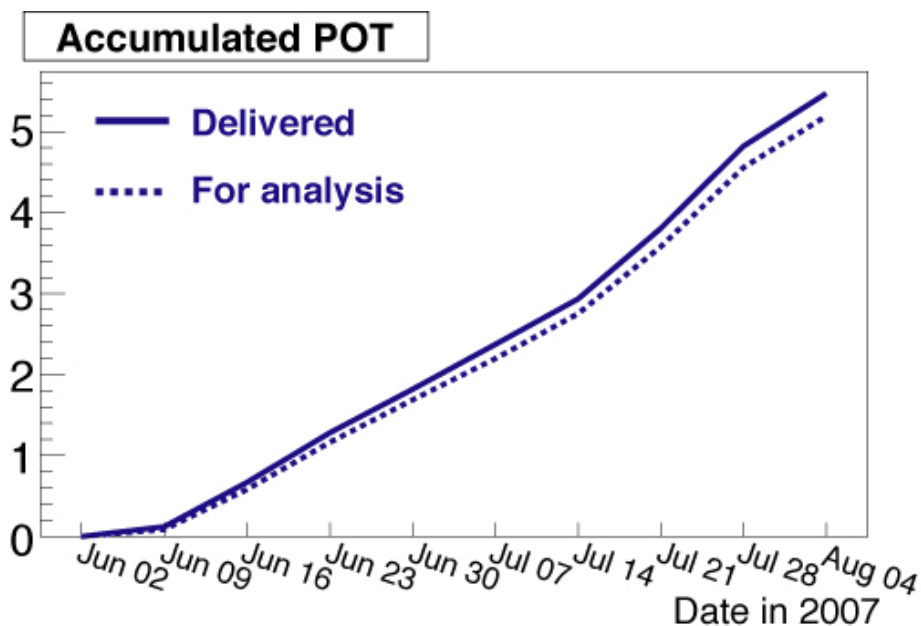

Figure 13. Accumulated protons on target (POT) for the SciBooNE FY 2007 beam run (antineutrino mode). The solid curve shows the total accumulated POT and the dashed curve shows the collected POT after beam data quality cuts.

During the FY 2007 beam run, SciBar had only four dead channels out of 14,336. The average efficiency for cosmic muons in SciBar, measured with cosmic data, is $99.85 \%$. The average light collected for one minimum ionizing particle traversing a SciBar cell horizontally (1.3 cm path length) is 20 photoelectrons. The average time resolution is $1.6 \mathrm{~ns}$. Preliminary simple cuts on clusters of hits in space and time can be used to find charged-current (CC) neutrino interactions. Applying these cuts to the FY 2007 beam run data yields $\sim 5$ events per $4 \times 10^{16}$ POT, for a total of approximately 5000 events in the 10.6 ton fiducial volume of SciBar. Over the eight weeks of the FY 2007 beam run, the number of events passing the simple CC cuts per POT was stable within 10\%. Figure 14 shows the time of the hits in the vertices from the CC candidate tracks; a clear square peak of beam induced neutrino events can be seen above the cosmic-muon background. 


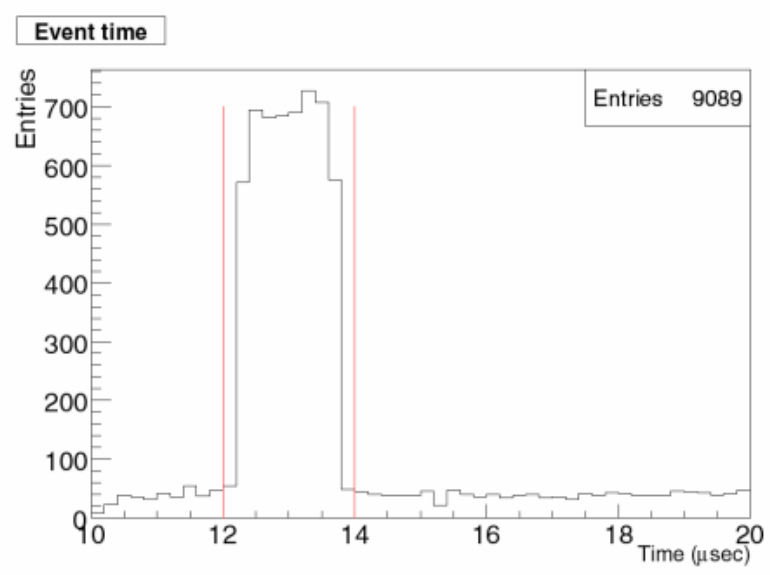

Figure 14. Recorded time of SciBar track vertices originating in the fiducial volume. The red lines denote a $2 \mu$ s window around the $1.6 \mu$ s beam spill.

All 256 EC channels were alive and performed within specifications during the FY 2007 beam run. The average efficiency for muons, measured independently with cosmic and beam data, is approximately $96 \%$ per plane. The inefficiencies are due mainly to gaps between the calorimeter modules. Figure 15 shows the calibrated energy deposited, in $\mathrm{MeV}$, by throughgoing cosmic muons on both the horizontal and vertical planes of the EC.

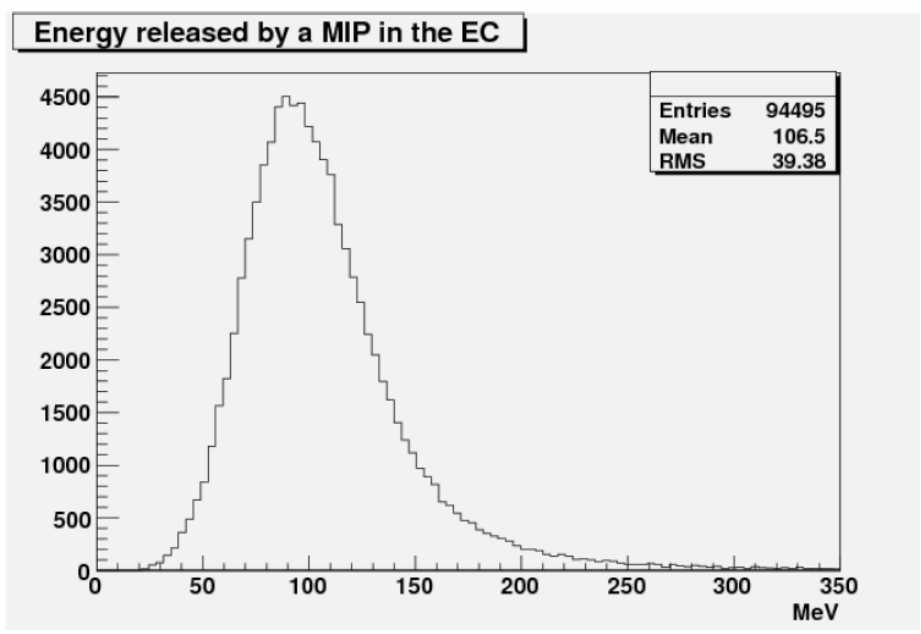

Figure 15. Energy deposited by cosmic muons in the horizontal and vertical layers of the EC.

The MRD had six dead channels out of 362 during SciBooNE's FY 2007 beam run. The average efficiency for muons, measured with cosmic-muon data was $99 \%$. As with the EC, much of the inefficiency stems from the gaps between the counters. Simple cuts on clusters of hits in the MRD yield approximately 10 events per $4 \times 10^{16}$ POT within the 19-ton fiducial volume of the MRD, for a total of over 10,000 neutrino interactions in the MRD over the duration of FY 2007 beam run. Figure 16 shows the number of events per week in the MRD 
passing the simple neutrino cuts, and the number of accumulated POT per week; the number of events in the MRD follows closely the number of accumulated POT each week.

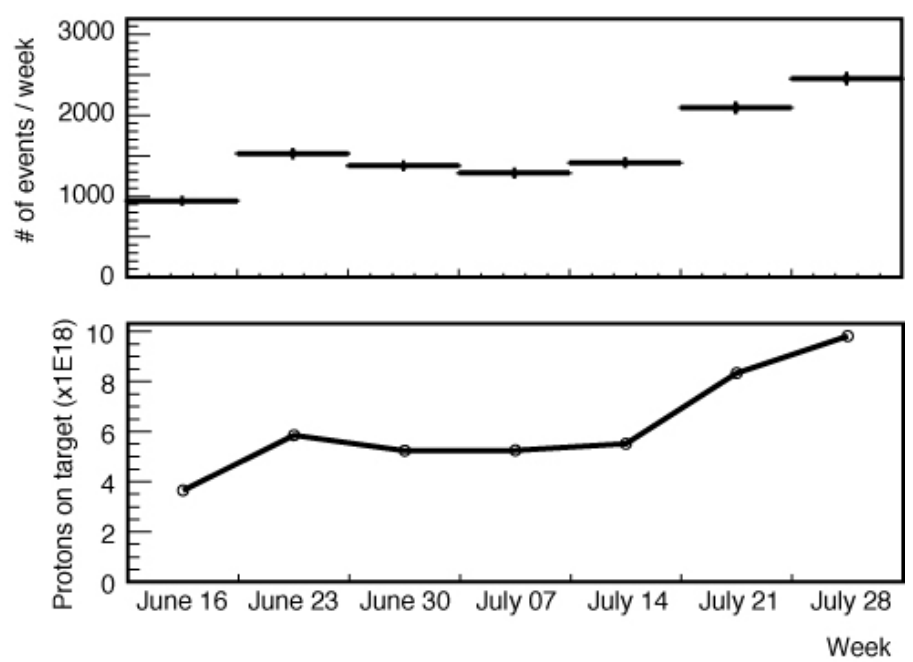

Figure 16. Progress of the SciBooNE MRD beam data collection. The top panel shows the number of events passing the simple neutrino cuts per week. The bottom panel shows the weekly POT count.

During the accelerator long shutdown of 2007, the polarity of the horn was switched back to neutrino mode. The experiment will record $1 \times 10^{20}$ POT in neutrino mode before switching back to antineutrino mode for the remainder of the FY 2008 run. All of the dead channels in the MRD and all but one in SciBar were replaced during the shutdown. Additionally, one plane of MRD counters was modified to improve the ADC resolution in the PMTs. SciBooNE will begin Run 2 with only one dead channel (out of 14,336+256+362) in the detector.

Fixed-Target Switchyard $120 \mathrm{GeV}$ (SY120) and MTest (R. Coleman, W. Kissel, E. Ramberg)

\section{Introduction to MI Beam Extraction for SY120}

The test beam originates from the resonant extraction of at least one Booster batch inside the Main Injector (MI). This batch usually consists of 60-80 RF 'buckets', with buckets separated by $19 \mathrm{nsec}$. Thus the batch is about 1.5 microseconds long. The full circumference of the MI is about 10 microseconds. It is possible to run with up to five Booster batches in the MI for MTest use. The batch or batches are accelerated to $120 \mathrm{GeV}$, circulate around the MI, and are slowly extracted over a macroscopic slow spill. For most usage of the test beam, there will be less than $100 \mathrm{kHz}$ of beam. If beam were smoothly extracted, this would imply that at most 1 particle per rotation of the MI batch will occur. The beam is not that smooth and there is up to 35\% double occupancy per MI rotation.

The length and duty cycle of the spill is determined by the Accelerator Division (AD), with guidance from the Office of Program Planning. For FY 2007 there was a single 3.6 second long 
spill per minute, for a maximum of 12 hours per day. The AD is implementing the ability to switch simply from this 3.6 second spill to a 1.0 second spill. This shorter spill could then be delivered more frequently for commissioning purposes and for those groups who are dataacquisition buffer limited.

The beam has an approximately 3\% momentum spread and can be focused to a $7 \mathrm{~mm} \mathrm{rms}$ spot size for $120 \mathrm{GeV}$ protons and approximately $2-5 \mathrm{~cm}$ rms spot for the lower momenta.

\section{$\underline{\text { SY120 in FY } 2007}$}

The number of SY120 pulses with beam in FY 2007 was 29,900. During FY 2007, time was also taken to rebuild the MTest beamline to optimize the low-energy particle production. ILC detector groups were particularly interested in low-energy pion beams capable of going as low as $1 \mathrm{GeV}$. The majority of the installation was completed in December 2006 and the first beam commissioning began in January 2007. User data-taking was supported as early as February and operations switched back and forth many times between installation and running for the users.

The changes to the MTest beam included the installation of a new movable target in the M04 enclosure. Accompanying this, the External Beams Group oversaw the installation of 11 new beamline elements, the movement of 13 magnets, and the movement of the entire beamline transversely in the tunnel. Also, special attention was given to minimizing the amount of scattering material in the beamline. Low-current power supplies and Hall probes for the dipoles were installed. This new beamline resulted in a vastly superior ability to deliver low-energy beam to the user areas. The resulting beam rates are given in Table 4 .

Table 4. Rate of beam delivered to the MT6 user facility for $1 \times 10^{11}$ protons in the Main Injector. Identification of electrons is by lead glass calorimetry.

\begin{tabular}{|c|c|c|c|c|}
\hline $\begin{array}{c}\text { Beam Energy } \\
(\mathrm{GeV})\end{array}$ & $\begin{array}{c}\text { Rate at Entrance to } \\
\text { MT6 (per spill) }\end{array}$ & $\begin{array}{c}\text { Rate at Exit of } \\
\text { MT6 (per spill) }\end{array}$ & $\begin{array}{c}\text { \%Pions, Muons } \\
\text { at exit of MT6 }\end{array}$ & $\begin{array}{c}\text { \% Electrons at } \\
\text { exit of MT6 }\end{array}$ \\
\hline 16 & 132,000 & 95,000 & $82 \%$ & $18 \%$ \\
\hline 8 & 89,000 & 65,000 & $42 \%$ & $58 \%$ \\
\hline 4 & 56,000 & 31,000 & $26 \%$ & $72 \%$ \\
\hline 2 & 68,000 & 28,000 & $34 \%$ & $65 \%$ \\
\hline 1 & 69,000 & 21,000 & $<50 \%$ & $>50 \%$ \\
\hline
\end{tabular}

The rates measured in the new beamline matched expectations and the new secondary beam is much easier to operate, also due to the improvements. The beamline operated at the following momenta: $120 \mathrm{GeV} / \mathrm{c}, 66 \mathrm{GeV} / \mathrm{c}, 32 \mathrm{GeV} / \mathrm{c}, 16 \mathrm{GeV} / \mathrm{c}, 8 \mathrm{GeV} / \mathrm{c}, 4 \mathrm{GeV} / \mathrm{c}, 2 \mathrm{GeV} / \mathrm{c}$ and $1 \mathrm{GeV} / \mathrm{c}$. The primary proton intensity was changed by varying the number of turns and Booster bunches (from 1 turn, 5 bunches to 2 turns, 84 bunches).

\section{Upgrades to the User Facility}

The test beam user facility gives users from around the world an opportunity to test the performance of their particle detectors in a variety of particle beams. A plan view of the facility 
is shown in Figure 17. In addition to the upgrade of the beamline, in FY 2007 there was a significant upgrade to the user area infrastructure. These improvements include:

- New differential Cerenkov counter

- Time-of-flight detector system

- $\quad 4^{\text {th }}$ MWPC tracking station

- Addition of new conference/break room

- Epoxy painting of the MTest floor

- Complete reinstallation of cabling between the beamline and counting room.

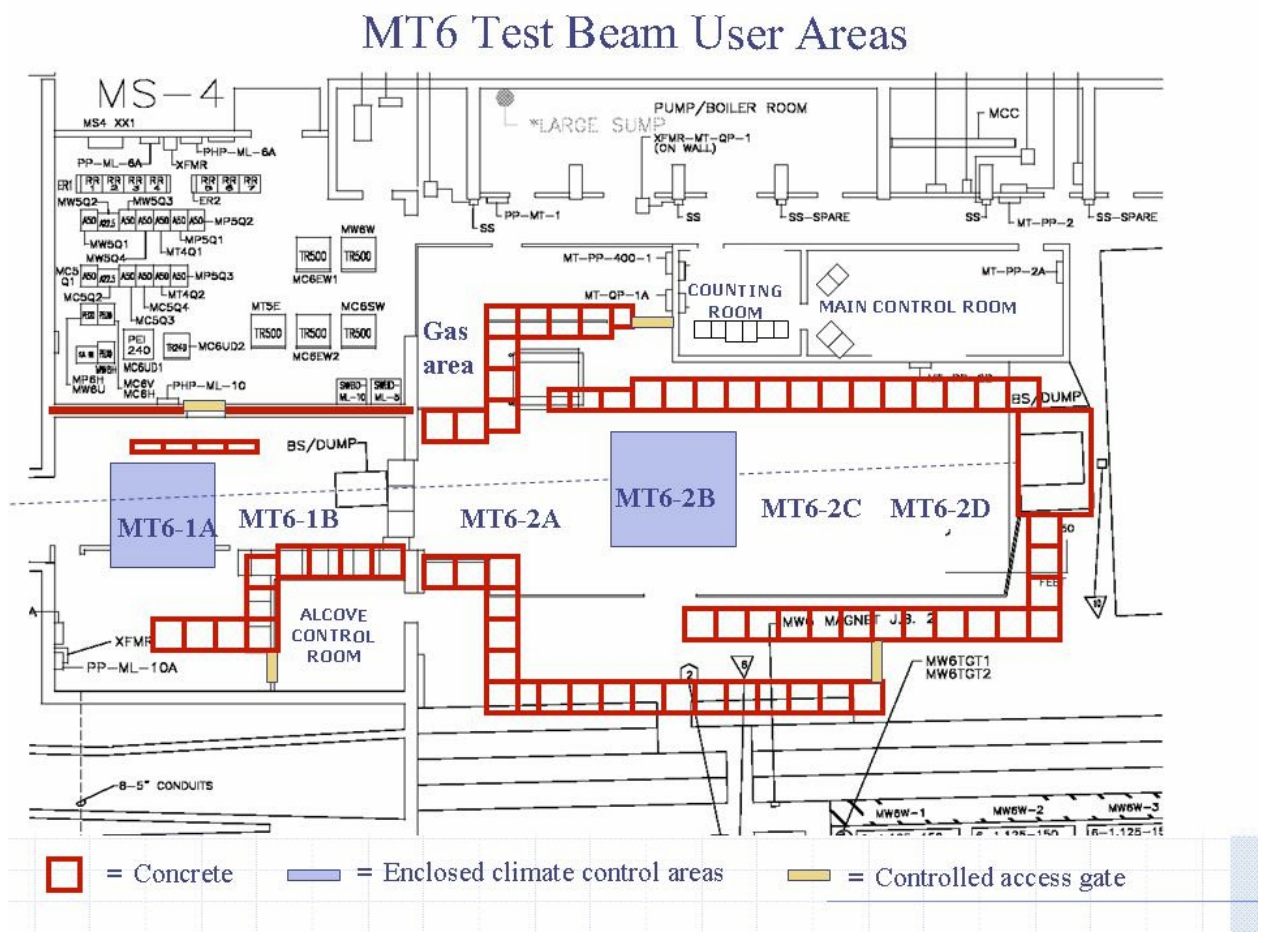

Figure 17. Plan view of the Meson Test Beam Facility.

\section{Research performed at the MTest Facility}

Each test beam experiment is required to prepare a Memorandum of Understanding with the Laboratory, in which beam, infrastructure, and safety requirements are spelled out in detail. Seven new experiments were approved and took data during FY 2007. An additional two experiments that took data in FY 2006 returned to take additional data in FY 2007. These nine experiments are listed in Table 5. Of the 22 weeks available between the startup of beam on February 21 and the beginning of the accelerator long shutdown on August 5, these test beam experiments took data for 14 weeks. 
Table 5. MTest experiments performed in FY 2007.

\begin{tabular}{|l|l|}
\hline Test & \multicolumn{1}{|c|}{ Description } \\
\hline & \\
\hline T-970 & DHCAL Detector Research \\
\hline T-968 & T2K Muon Monitor Prototype \\
\hline T-967 & Muon g-2 Calorimeter Test \\
\hline T-966 & Monolithic Pixel Detector for ILC \\
\hline T-965 & PSiP Photosensors Experiment \\
\hline T-964 & ILC GEM Chamber Characteristics \\
\hline T-963 & STAR Muon Telescope Detector \\
\hline T-958 & FP420 Fast Timing Test \\
\hline T-953 & U. Iowa Cerenkov Light Tests \\
\hline
\end{tabular}

The web site for the MTest facility can be found at http://www-ppd.fnal.gov/MTBF-w/. 